\title{
Disseminated cryptococcosis after H3N2 influenza in an immunocompetent child
}

\author{
Huan $\mathrm{Cao}^{1}$, Han Huang ${ }^{1}$, and Qianqian $\mathrm{He}^{1}$ \\ ${ }^{1}$ Children's Hospital Affiliated of Zhengzhou University
}

March 5, 2022

\begin{abstract}
A previous study has reported cryptococcal meningoencephalitis in a previously healthy adult affected with H1N1 influenza. Here, we report cryptococcosis occurred in a previously healthy child with H3N2-influenza, which further suggests a potential association between cryptococcosis and influenza viral infection. In this case, a 5-year-old girl presented with fever and cough was admitted to our hospital with positive test for H3N2 influenza and pulmonary infiltration by chest CT scan. After antibiotic regimens, the clinical symptoms were not improved at all. Her bone marrow culture proved Cryptococcus infection. Following treatments with amphotericin B liposome and flucytosine, her body temperature returned to normal. Pulmonary infection was completely absorbed following oral fluconazole treatment for 6 mon.
\end{abstract}

\section{INTRODUCTION}

Influenza results substantial mortality globally, one reason for which is co-infection. Influenza-associated pulmonary aspergillosis has been recognized gradually. However, other fungal infection secondary to influenza have been rarely reported. Here, we report cryptococcosis occurred in a child with H3N2-influenza, which may suggest a potential association between cryptococcosis and influenza.CASE REPORT

A previously healthy 5-year-old girl who was born and lived in Central China presented with fever for 20 days and cough for 18 days. Before referral to our hospital, chest CT scan showed bilateral pulmonary infiltrates; and throat swab test followed by RNA Detection PCR Assay showed positive for influenza viral infection at another hospital. The patient was treated with Oseltamivir at 45mg twice daily for 5 days. However, her fever and cough were not relieved at all.

After the patient was referred to our hospital, laboratory test results were as follows: H3N2 influenza viral infection by Rapid Multiplex PCR Method was positive; White blood cell count was $18.06 \mathrm{~K} / \mathrm{ml}$ with 72.4 \% neutrophils, $20.3 \%$ lymphocytes, $4.3 \%$ eosinophils, $2.8 \%$ monocytes, and $0.2 \%$ basophils; C-reactive protein (CRP) was $120.94 \mathrm{mg} / \mathrm{L}$ and Procalcitonin (PCT) $0.456 \mathrm{ng} / \mathrm{ml}$, and erythrocyte sedimentation rate (ESR) $120 \mathrm{~mm} / \mathrm{h}$; Tuberculin skin test and HIV test were negative.

Chest CT scan revealed progression of bilateral pulmonary infiltrates compared to the scan image taken 6 days ago (FIGURE 1). Additionally, CT scan showed new mediastinal lymphadenopathy. Head CT scan showed no significant abnormalities.

After treatments with Meropenem combined with Azithromycin and successive cefoperazone sulbactam combined with vancomycin, the patient's fever and cough were not relieved. Fiberoptic bronchoscopy revealed no special signs. Culture of bronchoalveolar lavage fluids was negative. Then marrow puncture and lumbar puncture were performed. Pressure of cerebrospinal fluids (CSF) was normal. Cerebrospinal fluid cytology, Chloride content, protein content, and the glucose level were all normal. Both acid-fast stain and ink stain of 
CSF were negative. And Cryptococcal antigen test was negative. Bone marrow culture revealed Cryptococcus neoformans. Assays of Cryptococcal antigen of bone marrow and blood performed were positive. Based on these results, pulmonary Cryptococcus and disseminated cryptococcosis were diagnosed. To evaluate cell immunity, lymphocytes and their subclasses were quantified. Results showed no significant decrease. After diagnosis, intravenous Amphotericin B liposome (AmBd) combined with intravenous flucytosine were given to the patient. The patient's body temperature gradually dropped to normal and cough was relieved after one week of anti- Cryptococcus regimen. The intravenous regimen was continued for a total of 3 weeks followed by a secondary oral fluconazole treatment for half a year. During this period of time, blood white cells, eosinophils, CRP, ESR of the patient gradually returned to normal (FIGURE 2). CT scans showed that the patchy shadow in the lung was fully absorbed.

\section{DISCUSSION}

Although histopathological examination was not performed, the diagnosis of pulmonary cryptococcosis and disseminated cryptococcosis made upon the symptoms of cough and fever, positive test for Cryptococcus capsular antigen, the results of bone marrow culture and chest CT scans, and the efficacy of anti-cryptococcosis treatment appears to be appropriate. Others have reported similar cases characterized by elevated blood white cells, C-response protein, erythrocyte sedimentation rate, eosinophils, and immunoglobin E which all gradually returned to normal after effective treatments[1].

In our case, bone marrow culture provided key evidence when pathogens from other biospecimens were not found. Induction therapy composed of AmBd plus flucytosine was recommended to continue for at least 4 weeks in previous healthy individuals with disseminated cryptococcosis according to the guideline in 2010[2]. In our case, induction therapy was shortened to 3 weeks because of fast alleviation of symptoms, remarkable absorption of chest CT, and concern of potential adverse effects of the medications. Oral fluconazole(6$8 \mathrm{mg} / \mathrm{kg}$ )after the induction therapy was given for 6 months. The child was followed up for half a year after discontinuing medication, and she was normal.

Cryptococcosis is more common in immunocompromised individuals, such as AIDs and organ transplantation recipients, than immunocompetent individuals[3]. Sites of infection include central nervous system, lung, and other organs. Disseminated cryptococcosis is rare in previous healthy children. The child in this case had no other known medical conditions, with negative HIV test and normal immunoglobin quantification and lymphocytes counts. However, the cryptococcosis in this case was combined with infection of H3N2 Influenza A. It is currently not clear whether a causal relation exists between two events.

Fungal infection concomitant or secondary to severe influenza have been reported in recent years, of which influence-associated pulmonary aspergillosis has been recognized gradually and valued clinically[4]. It may be related to the use of glucocorticoid, tracheal cannula, a long-term use of broad-spectrum antibiotics among others[4]. Influenza infection is an independent risk factor of aspergillosis[5]. In addition to aspergillus, other fungal infection secondary to severe influenza have been rarely reported[6]. Alireza et al[7] have reported Cryptococcal meningoencephalitis in an immunocompetent adult after H1N1 influenza infection. In our case, we are not certain which infection, influenza or cryptococcus, came first. A previous study indicated that a majority of healthy children were infected subclinically by Cryptococcus neoformans[8].

To this case, we speculate that the patient in the current case might be infected by cryptococcus intently before influenza. Cryptococcal infection compromised the immunity of the host, resulting in a transition from intent infection to dominant infection of cryptococcus. Alternatively, a prior influenza viral infection might predispose cryptococcosis. A previous study supports the latter hypothesis[9]. In the experiment, mice with influenza infection showed infiltration of neutrophils and macrophages, increased secretion of interferon- $\alpha 4 / \beta$, and decreased interferon- $\gamma$ in the lung. These changes predisposed mice to cryptococcosis by impairing host immune response to subsequent cryptococcal infection.

Influenza is epidemic globally and result in substantial mortality. This case may suggest a potential association between cryptococcosis and influenza viral infection, and further studies are required to determine the relationship. 
1. Setianingrum F, Rautemaa-Richardson R, Denning DW: Pulmonary cryptococcosis: A review of pathobiology and clinical aspects . Med Mycol 2019, 57 (2):133-150.

2. Perfect JR, Dismukes WE, Dromer F, Goldman DL, Graybill JR, Hamill RJ, Harrison TS, Larsen RA, Lortholary $\mathrm{O}$, Nguyen $\mathrm{MH}$ et al :Clinical practice guidelines for the management of cryptococcal disease: 2010 update by the infectious diseases society of america .Clin Infect Dis 2010, 50 (3):291322.

3. May RC, Stone NR, Wiesner DL, Bicanic T, Nielsen K:Cryptococcus: from environmental saprophyte to global pathogen . Nat Rev Microbiol 2016, 14 (2):106-117.

4. Verweij PE, Rijnders BJA, Bruggemann RJM, Azoulay E, Bassetti M, Blot S, Calandra T, Clancy CJ, Cornely OA, Chiller $\mathrm{T}$ et al :Review of influenza-associated pulmonary aspergillosis in ICU patients and proposal for a case definition: an expert opinion .Intensive Care Med 2020, 46 (8):1524-1535.

5. van de Veerdonk FL KE, Lestrade PP, Hodiamont CJ, Rijnders BJ, van Paassen J, Haas PJ, Oliveira Dos Santos C, Kampinga GA, Bergmans DC, van Dijk K, de Haan AF, van Dissel J, van der Hoeven HG, Verweij PE; Dutch Mycoses Study Group: Influenza-Associated Aspergillosis in Critically Ill Patients . $A M$ J RESP CRIT CARE 2017,196 (4):524-527.

6. Martin-Loeches I, M JS, Vincent JL, Alvarez-Lerma F, Bos LD, Sole-Violan J, Torres A, Rodriguez A: Increased incidence of co-infection in critically ill patients with influenza. Intensive Care Med 2017, 43 (1):48-58.

7. Alireza H, Alwyn R: Cryptococccal meningoencephalitis after H1N1 influenza . BMJ Case Rep 2012,2012 .

8. Goldma DL, Khin H, Abadi J, Lindenberg DJ, Pirofski L-a, Niang R, Casadevall A: Serologic evidence for Cryptococcus neoformans infection in early childhood . PEDIATRICS 2001,107 (5):e66.

9. Oliveira LVN, Costa MC, Magalhaes TFF, Bastos RW, Santos PC, Carneiro HCS, Ribeiro NQ, Ferreira GF, Ribeiro LS, Goncalves APF et al :Influenza A Virus as a Predisposing Factor for Cryptococcosis .Front Cell Infect Microbiol 2017, 7 :419.

10. Iuliano AD, Roguski KM, Chang HH, Muscatello DJ, Palekar R, Tempia S, Cohen C, Gran JM, Schanzer D, Cowling BJ et al :Estimates of global seasonal influenza-associated respiratory mortality: a modelling study . The Lancet 2018,391 (10127):1285-1300.

FIGURE 1, Computer tomography image of the case on the 21th day, 28th day of the course and after anti-Cryptococcus treatment. A, Amphotericin B liposome; F flucytosine; Flu, fluconazole;

FIGURE 2, Change of blood cells count and PCT, CRP. Meropenem and Azithromycin; C+V, cefoperazone sulbactam and vancomycin; A+F, Amphotericin B liposome and flucytosine; Flu, fluconazole;

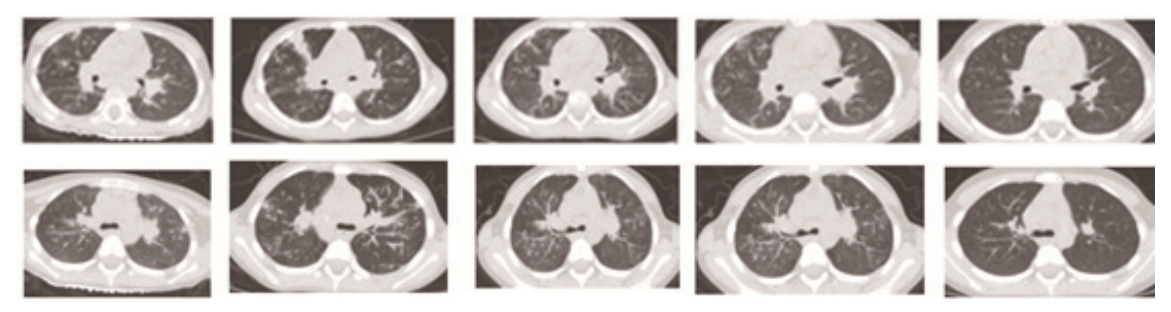




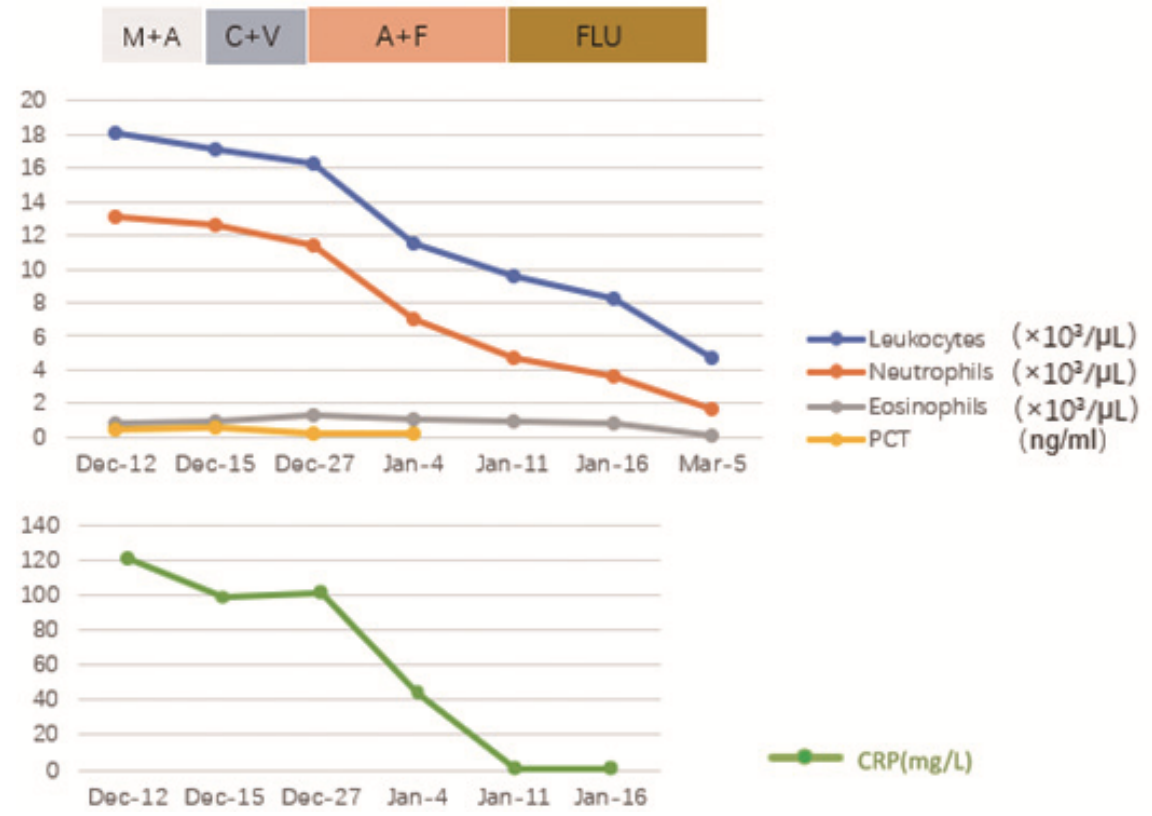

